Title : will be set by the publisher

Editors : will be set by the publisher

EAS Publications Series, Vol. ?, 2018

\title{
FUNDAMENTAL PARAMETERS OF MASSIVE STARS
}

\author{
Paul A. Crowther ${ }^{1}$
}

\begin{abstract}
We discuss the determination of fundamental parameters of 'normal' hot, massive OB-type stars, namely temperatures, luminosities, masses, gravities and surface abundances. We also present methods used to derive properties of stellar winds - mass-loss rates and wind velocities from early-type stars.
\end{abstract}

\section{Introduction}

The vast majority of stars in the Milky Way and external galaxies have masses close ' to that of the Sun, and live long ( $\geq$ Gyr), relatively peaceful lives. In contrast, stars with initial masses in excess of $\approx 10 M_{\odot}$, with O-type and early B-type spectral types on the main-sequence live for a relatively short life $(\sim 5-20 \mathrm{Myr})$, though have an astrophysical importance which belies their rarity. Massive stars and their ' evolved descendants possess powerful stellar winds, such that they are important contributors to the chemical and dynamical evolution of galaxies, due to their influence on the composition and energetics of the interstellar medium (ISM). Indeed, the primary origin of oxygen and other $\alpha$-elements in the universe is from massive stars. Ultimately, they end their life as a core-collapse Supernovae, causing 'further 'feedback' to the ISM.

Massive stars spend the majority of their lives in the blue part of the HertzsprungRussell (HR) diagram, and so emit the majority of their energy in the far- and extreme- ultraviolet. Consequently, in normal galaxies they are the dominant source of Lyman continuum photons. A typical O7 dwarf emits $10^{49}$ Lyman continuum photons every second for its main sequence life, and so OB stars ionize material in their vicinity, producing ionized nebulae, or H IIregions. Within the Milky Way, the Orion Nebula is the closest birth place of massive stars. Further afield, 30 Doradus, the Tarantula Nebula represents the greatest single concentration of massive stars within the Local Group of galaxies, containing many hundreds

\footnotetext{
1 Dept. of Physics \& Astronomy

University College London

Gower Street

London WC1E 6BT
} 
Title : will be set by the publisher

of OB stars within its Giant H IIregion. Further afield, starburst galaxies contain many so-called 'super-star clusters'. Massive stars are the only stellar objects to be observed in high redshift galaxies (e.g. MS 1512-cB58: Pettini et al. 2000)

It is clear that massive stars play a major role in astrophysics. In this article I shall explain how one can determine the fundamental properties of main sequence massive stars. In the following article, I shall discuss post-main sequence massive stars and their end states.

\section{Basic Concepts}

The principal measured parameter of an individual star is its brightness in the night sky, and its colour, namely the difference between magnitudes at two or more wavelengths. Historically, photography was the method of choice for astronomers. Since photography is most sensitive to visible radiation, spectral classification traditionally concentrated on these wavelengths. Only recently have electronic detectors, such as CCDs supplanted photographic plates.

Brightness, colour, and spectral types can lead to the luminosity and temperature of the star, the fundamental parameters to place it on the Hertzsprung-Russell diagram. The apparent magnitude of a star is typically measured through a filter, commonly the $V$ defined in the Johnson $U B V$ system. The absolute visual magnitude, $M_{V}$ is related to the apparent magnitude, $m_{V}$ via the relationship,

$$
M_{V}=m_{V}-5+5 \log d-A_{V}
$$

where $d$ is the distance (in parsec) and $A_{V}$ is the $V$-band extinction, defined by $A_{V}=R \times E(B-V)$, where $R \sim 3.1 . E(B-V)$ is merely the difference between the observed colour excess $m_{B}-m_{V}=B-V$ in the Johnson system and the intrinsic $(B-V)_{0}$ colour. According to Wien's law, most of the stellar radiation for luminous hot stars with their $\approx 30,000 \mathrm{~K}$ minimum $T_{\text {eff }}$ in emitted in the far UV regions. At optical wavelengths one is observing way out on the long wavelength tail of the spectral energy distribution (SED) for hot stars. In the $U B V$ system, intrinsic colors of $\mathrm{OB}$ stars are thus nearly degenerate (e.g., differing only by a few hundredths of a magnitude), typically $(B-V)_{0} \approx-0.3$ mag.

The highly wavelength dependent extinction of starlight by interstellar dust dominates the colors of luminous stars in the Milky Way. The observed optical colors of hot stars are used primarily to determine properties of the intervening interstellar medium and are not very useful to evaluate stellar parameters. Using the standard Galactic extinction curve of Seaton (1979), for every magnitude of extinction at the $V$-band, the far-UV extinction at $1200 \AA$ suffers four magnitudes, whilst only 0.4 magnitudes is suffered at $1 \mu \mathrm{m}$ and 0.1 magnitudes at $10 \mu \mathrm{m}$. Consequently, regions, such as the Galactic Centre which are invisible at UV and optical wavelengths are reasonably transparent longward of the $K$-band. Internal extinction in other galaxies differs somewhat from the Milky Way (Howarth 1983; Bouchet et al. 1985). In addition to continuous dust absorption, there are also discrete interstellar absorption features, principally the atomic Lyman HI 
and molecular $\mathrm{H}_{2}$ bands at UV wavelengths, whose strengths broadly scale with $E(B-V)$ (see e.g. Pellerin et al. 2002)

The $m_{V}$ measures the brightness of a star through a filter, but a luminosity is the emergent radiation over all wavelength bands. The Sun has an absolute visual magnitude of $M_{V}=4.82 \mathrm{mag}$, whilst typical luminous hot stars have $M_{V}=$ $-5 \mathrm{mag}$, so they are intrinsically of order 10,000 times brighter in the visual. Considering their output over all wavelengths, they exceed this by a further factor of 10 to 100. In magnitudes, one can write, $M_{\mathrm{bol}}=M_{V}+B . C$, where $M_{\mathrm{bol}}$ is the bolometric magnitude and B.C. is the bolometric correction. Thus latter quantity is strongly dependent on the $T_{\text {eff }}$, having an appreciable value for hot, blue stars. One can relate the luminosity of a star in solar luminosities to $M_{\mathrm{bol}}$ via

$$
M_{\mathrm{bol}}=-2.5 \log \left(L / L_{\odot}\right)+4.74
$$

where $L_{\odot}=3.845 \times 10^{33} \mathrm{erg} \mathrm{s}^{-1}$.

In principal, one obtains the luminosity of a star by measuring its $m_{V}$ and $(B-V)$, obtaining a distance, adopting a B.C. and utilizing the above equations together with a calibration relating the spectral type of the star to its intrinsic $B-V)_{0}$ colour. A $T_{\text {eff }}$ will first need to be obtained so that the B.C. can be determined. Various B.C.( $\left.T_{\text {eff }}\right)$ exist in the literature (e.g. Balona 1994). For O and early B stars, Vacca et al. (1996) derived

$$
\text { B.C. }=27.66-6.84 \log T_{\mathrm{eff}}
$$

The dependence is clearly very steep for $\mathrm{O}$ stars, so small errors in $T_{\text {eff }}$ may lead to large errors in luminosity. For example, the stellar luminosity of an O3 dwarf with absolute magnitude $M_{\mathrm{V}}=-5 \mathrm{mag}$ is $500,000 L_{\odot}$ if $T_{\text {eff }}=50,000 \mathrm{~K}$, but only $370,000 L_{\odot}$, if $T_{\text {eff }}=45,000 \mathrm{~K}$.

It is, of course, no trivial matter to determine the distance of an individual hot star, and this represents the greatest difficulty with deriving luminosities for Galactic OB stars. Due to their large distances, direct determination via stellar parallax methods are only possible for a dozen cases with Hipparcos (Lamers et al. 1997). Amongst the closest examples Hipparcos measured were $\zeta$ Oph (O9.5 Vn) and $\tau$ Sco (B0.2 IV) at distances of $\sim 135 \mathrm{pc}$, a hundred times more distant from the Sun than the closest star, $\alpha$ Centauri.

Within a decade, GAIA should enable reliable parallaxes to all optically visible Galactic OB stars. Until then, one has to resort to obtaining spectral type- $M_{\mathrm{V}}$ calibrations using OB stars in associations or clusters. Humphreys (1978) carried out a very extensive study for Galactic OB supergiants. This was extended to Magellanic Cloud associations by P. Massey and co-workers. These calibrations may then be applied to individual stars in the field. The advantage of Magellanic Cloud stars is that their distances are known to a precision better than $10 \%$. Consequently, despite their relative proximity, luminosities of Galactic OB stars are more imprecise than those of stars in the Magellanic Clouds or beyond. The limitation of extragalactic stars is principally that of spatial resolution. Fortunately, one finds reasonably good consistency between calibrations from Galactic 
Title : will be set by the publisher

and extra-galactic hot stars. Nevertheless, OB stars within a given spectral type show a substantial spread, which is thought to be typically \pm 0.5 mag.

One may also use radio observations to study the Galactic distribution of massive stars, since these are located in Giant H IIregions that are strong radio emitters (e.g. Smith et al. 1978). OB stars are confined to the thin disk of the Milky Way, and (crudely) map out the spiral arm structure (Georgelin \& Georgelin 1976). Knowledge of the ionizing fluxes of individual OB stars permits one to obtain total numbers of stars in H IIregions. The ionizing fluxes of hot stars is a very strong function of temperature and to a lesser degree surface gravity. Vacca et al. (1996) provided a $T$ eff calibration of Lyman $\left(Q_{0}\right)$ and He $\mathrm{I}\left(Q_{1}\right)$ ionizing fluxes for OB stars. The widely used $T_{\text {eff }}$-spectral type relation for OB stars adopted therein (see below) has been substantially revised recently, such that Crowther \& Dessart (1998) provide a calibration of $Q_{0}\left(T_{\text {eff }}, \log L\right)$, whilst Hubeny \& Lanz (2003) have recently produced a comprehensive tabulation of ionizing fluxes for dwarf O stars at a range of metallicities and gravities.

\section{Spectral Types}

The emergent continuum radiation from stars arises from the stellar photosphere while the absorption line spectrum comes from the atmosphere. In normal stars these regions overlap, can be treated as plane parallel, and are closely coupled to the stellar parameters.

Numerical subtypes distinguish differences within the spectral letters and go from number 0 to 9 (but with a few 0.5 divisions). Line widths and other line ratios are used to determine the atmospheric pressures (related to gas densities, local gravity, hence luminosity). These labels utilize roman numerals I-V, with the latter corresponding to the faintest luminosity class. While five luminosity classes are easily distinguished among cool $\mathrm{K}$ and $\mathrm{M}$ stars with their wide dispersion in $M_{V}$, only I, III, and $\mathrm{V}$ are adopted for $\mathrm{O}$ type stars which have a more limited $M_{V}$ range.

The most common system of spectral classification builds upon that of Morgan et al. (1943-MK) in which moderate spectral resolution is used to define natural groups of stars with similar spectral characteristics. This two dimensional system uses selected letters and numbers for the spectral types and subtypes, and roman numerals for the luminosity classes. Standard stars are typically selected for each spectral type and luminosity class. Classification of other stars is then carried out by obtaining their spectra and by using the spectral criteria an eye comparison to the standards. The dividing line for luminous hot stars at $T_{\text {eff }}$ just below $30000 \mathrm{~K}$ corresponds roughly to spectral type B1 on the Main Sequence. Thus O-early B spectral types are going to be the stars whose evolution we will follow in this article.

The MK system for these stars was modified and defined by Walborn (1971a). This was based upon spectra taken in the blue spectral region, on photographic plates, with a (reciprocal) dispersion of $63 \AA / \mathrm{mm}$. His classification system ranged from O4 through B2.5. Walborn (1971b) next identified a group of early O stars 
near $\eta$ Carina, which extended his spectral subtype system in a natural way to O3. The classification for O-early B type stars utilizes He I and He II line ratios, along with Si II, Si III and Si IV features. The leading lines are as follows: He I: $\lambda 4471$ and 4387 (all units here are $\AA$ ); He II: $\lambda 4542$ and 4686; Si II: $\lambda 4128-30$; Si III: $\lambda 4552$; Si IV $\lambda 4089$ and 4116. Basically, the appearance of the $\lambda 4542$ He II line distinguishes the hotter $\mathrm{O}$ from the cooler $\mathrm{B}$ spectral types. The $\mathrm{O}$ subtypes are defined by the ratio $\lambda 4471 \mathrm{He} \mathrm{I} / \lambda 4542 \mathrm{He}$ II which monotonically decreases towards earlier spectral type, such that the $\lambda 4471$ line is very weak, or totally absent at O3. In luminosity $\mathrm{V}$ class the $\lambda 4686 \mathrm{He}$ II line behaves similarly to $\lambda 4542$ although it is a bit stronger and it persists into the earliest B type stars.

For B spectral types, $\lambda 4542 \mathrm{He} I$ is absent and the He I lines are strong. Si IV lines, which are seen throughout the $\mathrm{O}$ types, begin to fade at B0. Si III lines appear and the Si III/Si,IV line ratio is useful between B0 and B1.5. At B2 and later subtypes, $\mathrm{Si}$ II appears as $\mathrm{Si}$ III begins to fade and the $\mathrm{Si}$ II/Si II ratio comes into use. In B and late-O type stars, the strength of these silicon features is luminosity dependent, being stronger in brighter stars. The helium lines, however, are less effected so that various silicon/helium line ratios are used as luminosity criteria. Among late-O-early-B stars, luminosity classes of Ia, Ib, III and V are recognized.

In most $\mathrm{O}$ type stars, though, a vastly different phenomenon begins to become apparent in the optical: the appearance of emission lines. Plaskett \& Pearce (1931) noticed that in some $\mathrm{O}$ stars, the $\lambda 4634-41 \mathrm{~N}$ III triplet came into emission along with $\lambda 4686$ He II. These stars they labelled Of type. Walborn (1971a) pointed out that most $\mathrm{O}$ stars he observed showed the N III lines in emission even though $\lambda 4686$ remained in absorption or was not present. He labeled these as $\mathrm{O}((\mathrm{f}))$ and $\mathrm{O}(\mathrm{f})$ subtypes, respectively. We know now from detailed line modeling in O star atmospheres (e.g., Mihalas et al 1972) that N III emission is a result of a fortuitous dielectronic recombination and implies nothing about the luminosity. On the other hand, the appearance of the $\lambda 4686 \mathrm{He}$ II line, whether in absorption, missing, or emission does say something about the stellar wind structure (Auer \& Mihalas 1972) and the luminosity. The status of $\lambda 4686 \mathrm{He}$ II is useful as a luminosity criterion. Following Walborn (1971a) the luminosity class of all but the latest type $\mathrm{O}$ stars is as follows: if the line is in absorption, the type is $\mathrm{OV}$; if missing, O III; and if in emission, Of. The ((f)) and (f) nomenclature is (almost) redundant as nearly every $\mathrm{O}$ star, aside from the latest types, has $\lambda 4640 \mathrm{~N}$ III in emission.

Recently, the O2 spectral type has been added by Walborn et al. (2002a), such that O2-4 stars are defined by the ratio of emission lines at $\lambda 4058 \mathrm{~N}$ IV and $\lambda 4634-41 \mathrm{~N} \mathrm{III}$, in contrast with the usual He absorption line criteria for OB stars. The principal motivation for this was that all O stars for which 4471 He I was very weak or absent were previously grouped together as O3, yet exhibited a range of spectral morphologies, and stellar temperatures from quantitative analysis. The most massive, hottest stars known, with N IV $\gg$ N III emission, are re-defined as O2 stars, and include HD 93129A (O2 If*) in the Carina Nebula. Until recently, this was thought to represent one of the most massive stars in the Milky Way 
Title : will be set by the publisher

(Taresch et al. 1997) but it has recently been found to be a binary (Walborn 2003).

The MK and Walborn classification systems depend on the spectra of stars in the vicinity of the sun. These all have a composition similar to each other and to the sun itself. How would one classify stars that had a substantially different abundance? For O stars this is not a significant problem since the spectral criteria involve hydrogen and helium lines for the most part. Silicon ions do play a role in classification in the late $\mathrm{O}$-early $\mathrm{B}$ stars and caution must be exercised in the cases of low metal abundance. For the LMC, with metal deficiencies of a factor 2 or 3 , the effects on the classification are barely noticeable. For the SMC, with an abundance down by a factor between 5 and 10, the silicon deficiency is obvious, but the silicon ion line ratios can be used with some confidence. Fortunately, the luminosity of these stars is known from their membership in the Magellanic Clouds and does not need to be established from their spectra. Lennon (1997) provides a revision to the spectral classification of B supergiants in the SMC.

Walborn (1976) reviewed and clarified the observations that certain O-early B stars had anomalously strong nitrogen or carbon lines, but otherwise normal features. These he had labelled as OBN or OBC stars. The OBC stars were also notable for having weak nitrogen lines, but the OBN stars have more or less normal appearing carbon features. OBN stars are found among both main sequence stars and supergiants, while the $\mathrm{OBC}$ stars are exclusive to giants and supergiants. Other symbol or small letter suffixes have been introduced for anomalous OB spectra. These will be discussed in detail in the following article. A review of optical classification criteria for O-type stars is given by van der Hucht (1996).

In addition to optical spectral classifications, it is also possible to assign, somewhat cruder, ultraviolet or near-IR classifications. Detailed atlases of $1150-1800 \AA$ ultraviolet $\mathrm{OB}$ star spectra taken with the $I U E$ satellite have been compiled by $\mathrm{N}$. Walborn and collaborators. These have recently been supplemented with far-UV

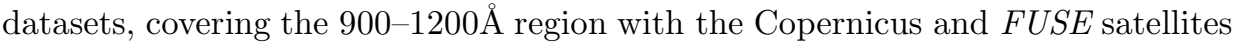
(Walborn et al. 2002b). Most recently of all, it has become possible to obtain classification quality spectroscopy of OB stars in the near-IR, specifically the $K$-band at $2 \mu \mathrm{m}$, which was carried out in a pioneering study by Hanson et al. (1996). This

has proved to be an extremely valuable resource by which we can study individual hot stars in regions that are inaccessible to optical or UV observations.

\section{Stellar temperatures}

Stellar temperatures of early-type stars, essential for subsequent determinations of radii and luminosities, are derived from a comparison between observed photometry or spectroscopy and models.

\subsection{Continuum techniques using LTE models}

The simplest case to consider is that for mid- to late- B dwarfs, with intermediate masses, which can be analysed using continuum energy distributions, via low dis- 
persion spectroscopy or photometry. Strömgren $u v b y \beta$ photometry, coupled with Kurucz LTE line blanketed model atmospheres, provides a powerful technique for the determination of $T_{\text {eff }}$ in B-type and cooler stars via the Balmer discontinuity.

Local Thermodynamic Equilibrium (LTE) Kurucz model atmospheres account very thoroughly for metal line blanketing and are widely employed for both earlyand late- type stars. Although a detailed discussion of stellar atmospheres is beyond the scope of this book, line blanketing is the influence of thousands to millions of bound-bound spectral lines on the atmospheric structure. LTE means that the ionization state of the gas and the populations of the atomic levels can be obtained from the local $T_{e}$ and $n_{e}$ via the Saha-Boltzmann distribution, such that collisional processes occur faster than radiative processes.

However, in the case of early- B dwarfs, B supergiants and O stars, several complications occur. Fatally for an approach based on the Balmer jump, this feature disappears for stars with temperatures in excess of approximately $30,000 \mathrm{~K}$. Although the intrinsic UV-optical-IR spectral energy distribution does differ between $\mathrm{B} 0$ and $\mathrm{O} 2-3$ stars, the effect is very subtle, and masked by uncertainties in interstellar reddening. In the case of B supergiants, the role of the Balmer jump is weakened, given that assumed solar abundances may no longer be appropriate. Consequently, the temperatures of such stars are derived via analysis of their line spectra, generally optical photospheric lines of helium (O-type) or silicon (B stars).

\subsection{Line techniques}

Generally, radiative processes generally dominate over collisional processes in hot star winds, so it is necessary to solve the equations of statistical equilibrium everywhere, i.e. non-LTE. The problem with this is that a determination of populations uses rates which are functions of the radiation field, itself is a function of the populations. Consequently, it is necessary to solve for the radiation field and populations simultaneously, which is computationally demanding, and requires an numerical iterative scheme to obtain consistency. Unfortunately, the problem is too complex for analytical solutions. Considerable effort has gone into developing realistic non-LTE model atmospheres for early-type stars in recent years by a number of independent groups. Unfortunately, consistently treating metal 'line blanketing' in extended non-LTE atmospheres is computationally demanding. Consequently, the earliest attempts by D. Mihalas in the late 1960's suffered from simplifications that only became surmountable two decades later, at which time large amounts of atomic data became available from the Opacity Project which was led by M. Seaton.

From such techniques, temperatures of hot stars are obtained using ionization equilibrium techniques, i.e. from suitable diagnostic lines of adjacent ionization stages. Naturally, determinations of $T_{\text {eff }}$ from model atmospheres are critically dependent on assumptions going into those models and the accuracy with which

surface gravities and elemental abundance ratios are determined. The majority of detailed analyses of OB stars have been subject to the assumption of hydrostatic equilibrium in a so-called 'plane-parallel' approach (e.g. Herrero et al. 1992; Puls 

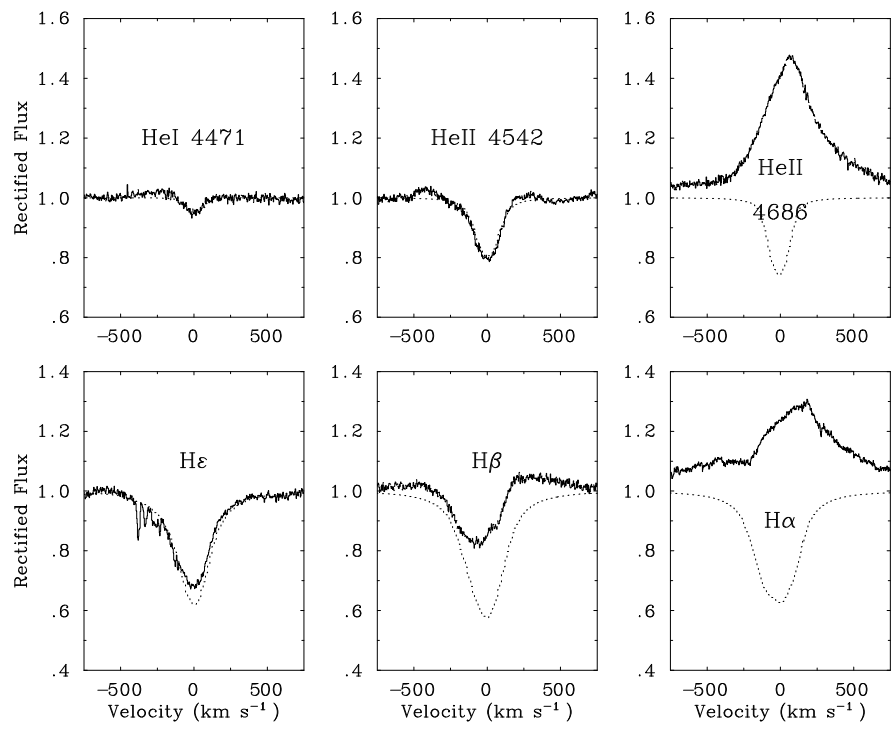

Fig. 1. Comparison between optical line profiles of HDE 269698 (O4Iaf+) and synthetic spectra (dotted) calculated using an unblanketed, plane-parallel version of TLUSTY with $T_{\text {eff }}=46.5 \mathrm{kK}, \log L / L_{\odot}=6.25, \log g=3.7$ (Crowther et al. 2002)
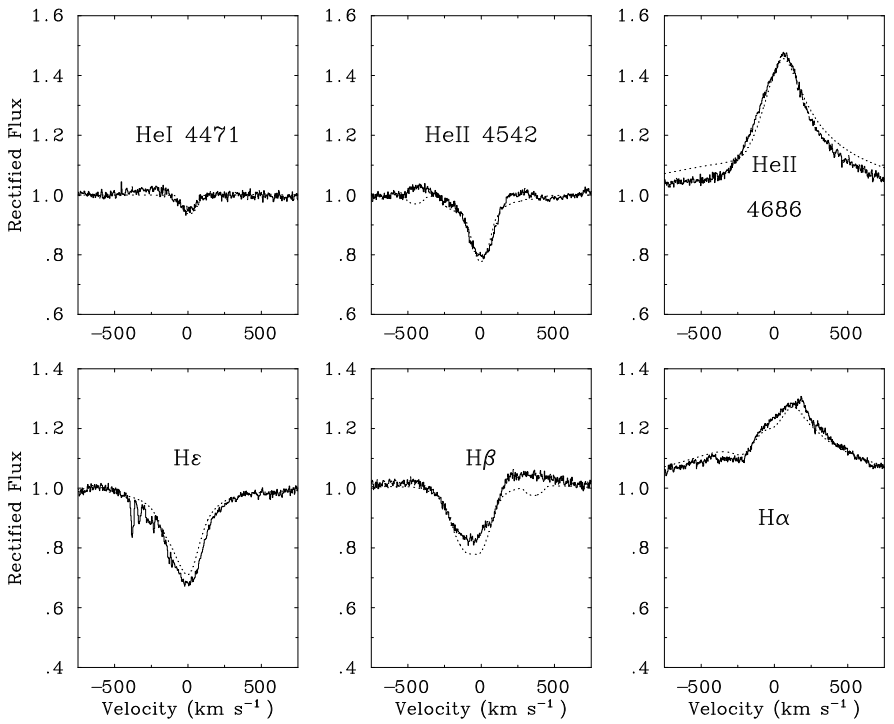

Fig. 2. Comparison between optical line profiles of HDE 269698 (O4Iaf+) and synthetic spectra (dotted) calculated using the line blanketed, spherical code CMFGEN with $T_{\text {eff }}=40 \mathrm{kK}, \log L / L_{\odot}=5.98, \log g=3.6, \dot{M}=8.5 \times 10^{-6} M_{\odot} \mathrm{yr}^{-1}, \beta=1, v_{\infty}=1750$ $\mathrm{km} \mathrm{s}^{-1}$ (Crowther et al. 2002) 
et al. 1996), where the spherical extension of the star is neglected. Optical He I-II lines generally provide temperature diagnostics for O stars (e.g. Herrero et al. 1992) while Si II-IV lines are used for B stars (e.g. McErlean et al., 1999). The typical range of temperatures spanned by $\mathrm{O}$ stars is $T_{\text {eff }} \simeq 50,000 \mathrm{~K}$ at the earliest subtypes to $T_{\text {eff }} \simeq 30,000 \mathrm{~K}$ at $\mathrm{B} 0 \mathrm{~V}$, and $T_{\text {eff }} \simeq 10,000 \mathrm{~K}$ at A0 V.

The temperature scale of OB dwarfs and supergiants has been compiled by various authors, most recently by Vacca et al. (1996). However, a word of caution is necessary at this point. This, and all previous compilations are based on plane-parallel pure H-He models. The effect of line blanketing from millions of metallic lines on the emergent spectra and ionization balance was neglected in most literature papers.

Within the past few years several non-LTE model atmosphere codes allowing for metal line blanketing have been developed. TLUSTY represents the current state-of-the-art for 'plane-parallel' studies of OB dwarfs (Hubeny \& Lanz 2003), whilst spherically extended non-LTE line blanketed codes include CMFGEN (Hillier \& Miller 1998), WM-BASIC (Pauldrach et al. 2001), FASTwIND (Santaloya-Rey et al. 1997) and the Potsdam code (Gräfener et al 2002). Recent studies of OB dwarfs have been carried out using CMFGEN and TLUSTY, employing metal line blanketing, resulting in systematically lower temperatures (e.g. Martins et al. 2002; Bouret et al. 2003).

The case for $\mathrm{O}$ supergiants is further affected by the presence of strong stellar winds. These may contaminate ('fill-in') photospheric absorption lines, causing temperatures to be overestimated still further in the standard plane-parallel assumption (Schaerer \& Schmutz 1994). Bohannan et al. (1990) demonstrated this for the O supergiant, $\zeta$ Pup (O4 If) in which wind blanketing reduced previous determinations by $>10 \%$. Putting stellar winds and line blanketing together can cause downward revisions in $T_{\text {eff }}$ by up to $20 \%$ in extreme OB supergiants (Crowther et al. 2002; Herrero et al. 2002). This is illustrated in Figures 1 and 2, which show respectively, spectral fits to HDE 269698 (O4 Iaf+) in the LMC using a unblanketed plane-parallel code, revealing $T_{\text {eff }}=46.5 \mathrm{kK}$, in which the wind lines are neglected, and blanketed, spherically extended code, revealing $T_{\text {eff }}=40 \mathrm{kK}$ plus simultaneous fits to wind features in the optical, UV and far-UV (Crowther et al. 2002). Such major downward revisions have a strong influence on the stellar luminosity of individual stars, which of course scale as $T_{\mathrm{eff}}^{4}$.

\section{Masses}

The mass of a star is perhaps its most fundamental property, yet it is generally very difficult to constrain for massive stars to better than a factor of two. This is because young massive stars of differing ages and masses cluster together very tightly in the upper left H-R diagram. There represent two, model dependent, methods that may be applied to derive a stellar mass, plus a third, more direct technique if the star happens to be a member of a double-lined, short period binary.

In general, massive stars are not members of double-lined, short period binaries. The primary method of deriving a star's mass is via a determination of the surface 


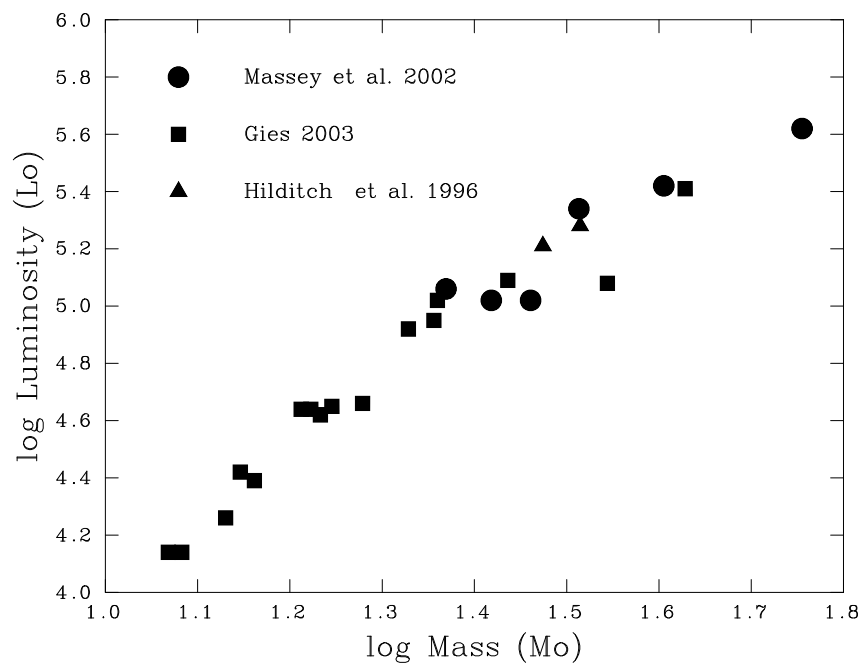

Fig. 3. Mass-luminosity relationship for young OB stars from binary orbits, taken from the Gies (2003) compilation, Hilditch et al. (1996) for DH Cep, plus Massey et al. (2002) for LMC stars

gravity from fitting line profile wings of Balmer hydrogen absorption lines. The spectroscopic mass is determined from this gravity determination, using

$$
g=\frac{G M}{R^{2}}
$$

In reality, an 'effective gravity' is determined for hot stars due to the complication of radiation pressure, as will be discussed later in this article, and is expressed as $\log g$ in cgs units. Typical values for OB dwarfs are $\log g=4-4.5$, with somewhat lower gravities, implying more extended atmospheres, for supergiants, e.g. $\log g \sim 3$ in late $\mathrm{O}$ or early $\mathrm{B}$ supergiants. High dispersion optical spectroscopy is needed in order to model the Balmer line wings accurately enough.

Alternatively, the position of a star in the H-R diagram can be compared to predictions from evolutionary tracks to extract a stellar mass. This is most straightforward for main sequence stars, as it is often uncertain whether individual OB supergiants are evolving to cooler temperatures immediately after the main sequence, or blueward after an excursion to the red part of the H-R diagram. Surface abundances will generally allow one to discriminate between these alternatives, but these are difficult to reliably measure for hot stars.

Herrero et al. (1992) first established a "mass discrepancy" between the sppectroscopic mass determinations and those resulting from comparison with evolutionary tracks. This remains to this day, with evolutionary masses up to a factor of two times higher than spectroscopic masses for OB stars. Potential problems lie in 
both the stellar luminosities obtained from model analyses, see above, and in the evolutionary models. For the latter, there was previously a rather tight correlation between luminosity and mass for hot stars, but the recent inclusion of rotation into evolutionary calculations has revealed that alternative initial rotational velocities can lead to a considerable spread in $\left(T_{\text {eff }}, \log L\right)$ for stars of identical initial mass.

The most accurate method of determining masses for OB stars, and so establishing which, if any, of these two approaches is correct, is if these are members of binary systems. Unfortunately, very few double-lined $\mathrm{O}+\mathrm{O}$ systems have sufficiently short orbits to permit spectroscopic monitoring and determination of complete light curves practical. Gies (2003) presents a summary of recent studies of detached (non-interacting) massive binaries. These permit a direct massluminosity relation for young OB stars, as shown in Fig. 3. Between $10-30 M_{\odot}$, $L \propto M^{2.5}$, above which $L \propto M^{1.75}$.

Massey et al. (2002) have used very massive binaries in the LMC R136 cluster to confirm evolutionary mass determinations, including a mass of $57 M_{\odot}$ for the O3 V primary of R136 \#38 in close agreement with $53 M_{\odot}$ from evolutionary models. Nevertheless, problems do remain in some cases. Consequently, the jury remains out for the present on this question. An added complication with the binary method is that stellar temperatures and bolometric corrections need to be derived or adopted from calibrations. Errors in such calibrations may exacerbate differences. Ultimately, the use of modern temperature calibrations in binary studies and line blanketed stellar atmosphere models will likely resolve evolutionary and spectroscopic masses for early-type stars (Herrero et al. 2002).

\section{Stellar Wind Properties}

Winds are ubiquitous amongst massive stars, although the physical processes involved depend upon the location of the star within the H-R diagram. Mass-loss crucially affects the evolution and fate of a massive star, while the momentum and energy expelled contribute to the dynamics and energetics of the ISM.

The existence of winds in O-type stars has been established since the 1960's, when the first rocket UV observations revealed the characteristic P Cygni signatures of mass-loss from C IV $\lambda 1548-51$, Si IV $\lambda 1393-1402$, and N v $\lambda 1238-42$ (Morton 1967). A theoretical framework for mass-loss in hot stars was developed by Castor, Abbott \& Klein (1975), known as CAK theory via line-driven radiation pressure. The fundamental characteristics of stellar winds are velocities and mass-loss rates. The former can be directly observed, whilst the latter relies of varying complexity of theoretical interpretation.

\subsection{Theory of radiatively driven winds}

The basic mechanism by which hot star winds are driven is the transfer of photospheric photon momentum to the stellar atmosphere through absorption by spectral lines. The velocity reached far from the star tends towards a terminal value, $v_{\infty}$, due to the geometrical dilution of the photospheric radiation field, whilst the 
mass-loss rate relates to the velocity field, $v(r)$ and density, $\rho(r)$ via the equation of continuity

$$
\dot{M}=4 \pi r^{2} \rho(r) v(r)
$$

for a spherical, stationary wind.

The properties of stellar winds depend on both the number of metal lines available to absorb photon momentum, and on their ability to absorb, i.e. their optical thickness. Analytical solutions involve force multiplier parameters, $\alpha, \delta$ and $k$ which characterize the outflow (Kudritzki et al. 1989). Of these, $\alpha$ controls the fraction of optically thick/thin lines, $k$ the number of strong lines, and $\delta$ the ionization balance.

It can be shown that,

$$
v_{\infty} \propto\left(\frac{\alpha}{1-\alpha}\right) v_{\mathrm{esc}}
$$

and

$$
\dot{M} \propto k^{1 /(\alpha-\delta)}
$$

From above, there is expected to be a close relationship between the terminal velocity and the escape velocity,

$$
v_{\mathrm{esc}}=\left(2 g_{\mathrm{eff}} R\right)^{0.5}
$$

where the effective gravity

$$
g_{\text {eff }}=\frac{G M}{R^{2}}(1-\Gamma)
$$

is the stellar gravity corrected for the reducing effect of radiation pressure (via Thompson scattering) on the gravitational potential via $\Gamma$ which is related to the stellar luminosity and mass. It is clear that the determination of stellar escape velocities depends on knowledge of stellar masses and radii.

Recent theoretical studies have indicated that CNO elements are principal line drivers for the outer, supersonic part of the wind, whilst iron group elements are responsible for the inner, subsonic part (Vink et al. 1999). The former determine the wind velocity, and the latter the mass-loss rate, at least for compositions close to Solar metallicity.

Hot stellar winds are predicted to depend on the metal content, $Z$, as follows. Puls et al. (1996) showed that $k \propto Z^{1-\alpha}$, such that Eqn 6.3 implies

$$
\dot{M} \propto Z^{(1-\alpha) /(\alpha-\delta)}
$$

Inserting typical values of $\alpha(=2 / 3)$ and $\delta(=1 / 10)$ suggests an exponent of $\sim 0.6$, close to 0.5 deduced by Kudritzki et al. (1987) and 0.7 derived by Vink et al. (2001). Since $\alpha$ is also predicted to be a function of metallicity, Leitherer et al. (1992) derived $v_{\infty} \propto Z^{0.13}$ for OB stars.

Puls et al. (1996) and Kudritzki et al. (1999) obtained a theoretical relationship between the bolometric luminosity of an early-type star and the wind momentum, $\dot{M} v_{\infty}$ modified by the square-root of the stellar radius, i.e. the socalled wind-momentum relation, as follows: 


$$
\dot{M} v_{\infty}\left(R / R_{\odot}\right)^{0.5} \propto L^{1 /(\alpha-\delta)}
$$

For O stars, taking $\alpha=0.6, \delta=0.05$, implies a luminosity exponent of $\sim 1.8$, close to that observed for Galactic O supergiants $(\sim 1.5)$ and dwarfs $(\sim 1.6)$ according to Kudritzki \& Puls (2000). The exponent for B supergiants is different, due to the change in ionization of the elements contributing to the radiative line acceleration. Differences in coefficient follow from the recent revision in temperature calibration for $\mathrm{O}$ and early B stars (Herrero et al. 2002). This relationship provides a potential independent method of determining distances to galaxies beyond the Local Group, although it is primarily of use for (less luminous) A supergiants which are visually the brightest hot stars in galaxies.

\subsection{Wind velocities}

Ultraviolet P Cygni profiles, ubiquitous in O-type stars provide a direct indication of stellar winds. Wind material approaching the observer within a column in front of the star is blueshifted by the Doppler effect. Scattering of radiation out of this direction causes a reduction in the observed flux on the blue side of the profile. From the regions on either side of this column wind material may scatter radiation towards the observer. This may occur from either the approaching (blue-shifted) or receding (red-shifted) hemisphere, the extra flux seen by the observer, originating from this scattered radiation, is a symmetric emission component on both sides of the profile. The overall effect is therefore, asymmetric with blue absorption and red emission. The wavelength of the blue edge of the absorption provides a measure of the asymptotic wind velocity. Accurate wind velocities of OB and W-R stars can be readily obtained in this way from HST or IUE observations of saturated C IV $\lambda 1548-51$, or Si IV $\lambda 1393-1402$ P Cygni profiles (Prinja et al. 1990; Lamers et al. 1995). The range in velocities encountered is considerable: $v_{\infty}=3500$ $\mathrm{km} \mathrm{s}^{-1}$, corresponding to $\geq 1 \%$ of the speed of light, in the earliest O stars, to 100 $\mathrm{km} \mathrm{s}^{-1}$ in some AB supergiants.

The number of OB stars observed in our Galaxy, principally with IUE, far exceeds that from external galaxies due to their relative UV brightness. Nevertheless, sufficient extragalactic hot stars have now been observed, principally with $H S T$, for us to be able to make useful comparisons. Wind velocities of LMC O stars differ little from Galactic counterparts (Garmany \& Conti 1985), while a more prominent effect is observed in the SMC, particularly amongst early O stars (Walborn et al. 1995; Prinja \& Crowther 1998).

Theoretically, wind velocities of hot stars are expected to follow a so-called $\beta$-law,

$$
v(r)=v_{\infty}\left(1-\frac{R}{r}\right)^{\beta}
$$

where $R$ is the stellar radius and $\beta \sim 0.8-1.5$. UV line profile modelling appears to confirm expectations. 
In spite of often uncertain stellar masses, Lamers et al. highlighted the socalled 'bi-stability' jump in wind properties around B 1 ( $\left.T_{\text {eff }} \sim 21,000 \mathrm{~K}\right)$, above which $v_{\infty} \simeq 2.65 v_{\mathrm{esc}}$, and $v_{\infty} \simeq 1.4 v_{\mathrm{esc}}$. This is thought to result from the change in ionization of the dominant elements contributing to the line force (Vink et al. 1999).

\subsection{Mass-loss rates}

Observationally, estimates of OB mass-loss rates are obtained from radio continuum fluxes, optical/IR line profiles, or UV wind lines. In extreme cases, $\dot{M}$ may exceed $10^{-5} M_{\odot} \mathrm{yr}^{-1}$ in some early $\mathrm{O}$ supergiants, but values 10-100 times lower are more typical.

\subsubsection{IR-radio continua}

Winds in hot stars can be readily observed at IR-mm-radio wavelengths via the free-free (Bremsstrahlung) 'thermal' excess caused by the stellar wind, under the assumption of homogeneity and spherical symmetry. Mass-loss rates can be determined via application of relatively simple analytical relations (e.g. Wright \& Barlow 1975) which reveal that the continuum flux, $S_{\nu} \propto \nu^{0.6}$, in a spherically symmetric, isothermal envelope which is expanding at constant velocity. The emergent flux is set by the distance to the star $d$, mass-loss rate and terminal velocity as follows

$$
S_{\nu} \propto\left(\frac{\dot{M}}{v_{\infty}}\right)^{4 / 3} \frac{\nu^{0.6}}{d^{2}}
$$

Other factors, including differences in composition, ionization balance, electron temperature generally play a minor role in normal OB stars. Barlow \& Cohen (1977) used this approach together with IR excess fluxes to determine mass-loss rates for a large sample of Galactic OBA supergiants. Radio measurements of OB stars were carried out by Abbott and collaborators with the VLA, as summarised by Bieging et al. (1989), and by Leitherer and collaborators with the Australia Telescope (Leitherer et al. 1995). Unfortunately, OB stars with relatively weak winds do not show a strong IR excess or radio flux, so mass-loss results from this technique have solely been for nearby hot stars with dense winds.

Collisions between stellar winds from stars in a binary system will cause nonthermal (synchrotron) radio emission, so care needs to be taken against overestimating mass-loss rates from (apparently) single stars in this way. Generally this is accomplished by carrying out observations at multiple radio frequencies.

\subsubsection{Optical and IR line profiles}

$\mathrm{H} \alpha$ has long been recognized as the prime source of mass-loss information in early-

type stars. However, accurate determinations rely upon a complex treatment of the lines and continua, i.e. non-LTE, spherically extended models which treat the 
sub- and supersonic atmospheric structure. Such techniques have already been touched upon with regard to surface temperatures of hot stars. Problems with using fits to $\mathrm{H} \alpha$ to derive mass-loss rates may include blending with He II $\lambda 6560$ in $\mathrm{O}$ stars, together with nebular contamination from $\mathrm{H}$ II regions, and broadening of the central emission component by stellar rotation.

Hot stars in the Magellanic Clouds provide us with the means to compare empirical mass-loss properties with predictions at lower $Z$. Although the number of stars analysed for the Magellanic Clouds remains small (Puls et al. 1996), the mass-loss rates of LMC and SMC O stars do appear to be lower than Galactic counterparts, in reasonable accord with theoretical predictions.

Although moderate to high spectral resolution observations of OB stars are rare at near-IR wavelengths, there exist wind diagnostics analogous lines to optical lines (Bohannan \& Crowther 1999). This provides the prospect of the determination of mass-loss properties for hot stars obscured at optical wavelengths via IR diagnostics.

\subsubsection{UV P Cygni profiles}

UV P Cygni profiles from metal resonance lines provide direct evidence for massloss in hot stars. Unfortunately, mass-loss determinations require knowledge of elemental abundances, the degree of ionization of the ion producing the line, and knowledge about the form of the velocity law.

Recent analyses of hot star winds via UV spectroscopy are generally carried out using the Sobolev with Exact Integration (SEI) method (Lamers et al. 1987; Haser et al. 1998), named after the pioneer of stellar wind studies, V. Sovolev from the late 1950's. In the SEI approach, UV P Cygni line profiles are fitted to reveal $\dot{M} q$, where $q$ is the, a priori unknown, fractional population of a particular ionization stage within a particular element. Direct ionization information is limited to observed ions from IUE or HST spectroscopy: Nv $\lambda 1238-42$, Si IV $\lambda 1393-1402$, and C IV $\lambda 1548-51$, so mass-loss rates may only be estimated via ionization balance information, generally from non-LTE atmospheric models. Far-UV FUSE observations complement previous observations, providing information on many more ionization stages, including N III $\lambda 989-91$, C III $\lambda 977$, S IV $\lambda 1062-72$, S VI $\lambda 933-44$, P V $\lambda 1118-28$. Massa et al. (2003) have used SEI modelling of line profiles measured FUSE spectroscopy of LMC OB stars to derive empirical ionization balances, albeit relying on theoretical mass-loss rates from Vink et a. (2001).

\subsection{Structure}

There is extensive observational evidence that hot star winds are not smooth, steady outflows. Intensive monitoring of unsaturated UV P Cygni lines with IUE led to extensive variability in the absorption components. To produce such variability, the wind structure must be on a large scale, covering a substantial fraction of the disk. In contrast, emission components of $\mathrm{P}$ Cygni profiles were found to be rather less variable, since it forms from a more global average of the wind. 
Subsequent optical studies have also led to absorption and emission line variability.

There is also indirect evidence that OB winds have a turbulent structure. Saturated P Cygni profiles have extended black troughs, which are thought to be a signature of a highly non-monotonic wind. Soft X-ray emission is also observed, which is thought to originate from embedded wind shocks (Chlebowski et al. 1989; Lucy \& White 1980). Such shocks are also thought to be the means by which high ionization stages are observed, most notably $\mathrm{N}_{\mathrm{V}} \lambda 1238-42$, in stars for which the $T_{\text {eff }}$ is too low from normal means. This is via a process known as super- (or Auger) ionization.

A likely explanation for this turbulent structure is theoretical evidence for a strong instability of line driving to small-scale velocity perturbations. There is a strong potential in line scattering to drive wind material with accelerations that greatly exceed the mean outward acceleration. Simulations demonstrate that this instability may lead naturally to a highly structured flow dominated by multiple shock compressions (e.g. Runacres \& Owocki 2002). Such predictions are broadly consistent with recent Chandra X-ray line spectroscopy of $\zeta$ Pup (Cassinelli et al. 2001) and other early O stars. Interpretation of the X-ray spectroscopy of $\theta^{1} \mathrm{C}$ Ori indicates a magnetic origin.

If hot star winds are structured, or clumped, it remains to be seen whether derived mass-loss rates of OB stars, as derived from optical or radio measurements, represent over-estimates of true values. Overall consistency between e.g. $\mathrm{H} \alpha$ and radio determinations (Lamers \& Leitherer 1993) is rather good, suggesting that if winds are highly clumped, they are similar on scales of $\leq 1.5 R_{*}(\mathrm{H} \alpha)$ and $\sim 1000$ $R_{*}$ (radio). Time-averaged line profiles of OB stars that have been intensively monitored do remain remarkably stable on scales of years. Nevertheless, recent FUSE spectroscopic observations of $\mathrm{O}$ supergiants in the Magellanic Clouds require that their winds are clumpy in order to simultaneously reproduce far-UV and optical wind lines, principally $\mathrm{P} \vee \lambda 1118-28$, unless phosphorus is underabundant relative to other elements in these galaxies (Crowther et al 2002). Evans et al. (2003) arrive at similar conclusions in late $\mathrm{O}$ and early B supergiants using S IV $\lambda 1062-72$. Although the ISM abundance of Phosphorus in the Magellanic Clouds is uncertain, Sulphur is well constrained. Consequently, the most credible possibility is that OB supergiants are clumped (see also Massa et al. 2003).

\section{Rotation}

During the formation process of all stars, including hot luminous OB stars, the conservation of angular momentum implies that they will commence their Main Sequence life rotating very fast, perhaps close to break-up velocity. Due to rotational broadening mechanisms, we are able to measure current OB rotational velocities from optical or UV photospheric line profiles. For OB stars, the principal compilations are by Conti \& Ebbets (1977) and Howarth et al. (1997).

Due to projection effects, $v \sin i$ is actually measured, where $i$ is the inclination of the system on the sky as viewed by the observer. In addition, measured projected rotation velocities underestimate the true projected equatorial velocity, 
$v_{e} \sin i$, due to limb darkening in rapid rotators (Collins \& Truax 1995). From a sample of over $400 \mathrm{OB}$ stars, Howarth et al. determined a median value of $v \sin i=$ $90 \mathrm{~km} \mathrm{~s}^{-1}$, which de-projects to a value $10 \%$ higher on average. The determination of rotational velocities is aided by some very slow rotators which serve as useful templates. $\tau$ Sco (B0.2 IV) is the most famous example, with $v \sin i<5 \mathrm{~km} \mathrm{~s}^{-1}$.

One finds that the O Main Sequence distribution is much broader than that of supergiants, with only 3 supergiants amongst 33 stars with $v_{r} \sin i>200 \mathrm{~km} \mathrm{~s}^{-1}$. This can be understood in that as a star evolves from the ZAMS, its rotational velocity will decrease due to conservation of angular momentum, lowering the limit to the distribution. The various distributions are summarised by Howarth et al. (1997).

Theoretically, Friend \& Abbott (1986) first considered the effect of centrifugal acceleration on hot star winds, extended by Bjorkman \& Cassinelli (1993) to allow for the azimuthal dependence, who found that $\dot{M}$ increases towards the equator, and $v_{\infty}$ decreases, leading to an oblate 'wind-compressed disk'. This has been widely applied to Be stars, a subset of B stars exhibiting emission lines. Subsequently, it was established by Owocki et al, (1996) that a quite different geometry may result, by additionally allowing for non-radial components of the line force, and the increased polar radiation flux via gravity darkening (von Zeipel 1924). Due to a reduced escape velocity, the equatorial velocity law is slower than at the pole, inhibiting the equatorial disk and the original scaling of mass-loss can be reversed, such that a prolate geometry results. By way of example, an early-B dwarf rotating at $85 \%$ of its critical, or break-up, velocity was found by Petrenz $\&$ Puls $(2000)$ to have a strongly prolate structure $\left(\rho_{\text {pole }} / \rho_{\text {eq }} \leq 15\right)$ with a polar (equatorial) terminal velocity of $1030(730) \mathrm{km} \mathrm{s}^{-1}$. The global mass-loss rate was not found to deviate from its 1D value by greater than 10-20\%, except for supergiants close to the Eddington limit.

\section{Composition}

The French philosopher Auguste Comte famously, and inaccurately, predicted of stars in 1835 that '.. we would never know how to study by any means their chemical composition ..' Fortunately, theoretical knowledge of stellar atmospheres, atomic data, together with high-resolution spectrograph's now permits abundance determinations for most normal stars, including hot, luminous OB stars.

This can only readily be carried out using optical photospheric lines of $\mathrm{H}$, He and metals, based on the same non-LTE techniques described above that are used to derive $T_{\text {eff }}, \log g$. Consequently, it is generally solely hydrogen-helium abundances which have been determined to date for a relatively large number of O stars (e.g. Herrero et al. 1992). Whilst most main sequence stars showed normal He abundances, $\mathrm{He} / \mathrm{H} \approx 0.1$ by number, as expected, rapid rotators were surprisingly found to have enriched He at their surfaces, suggesting that rotational mixing has brought products of core nucleosynthesis to the surface while the star is still on the main sequence. Advances in model atmosphere modelling since then have generally supported these results. Recently, Bouret et al. (2003) have 
analysed the CNO abundances of a sample of SMC O dwarfs using non-LTE line blanketed models.

Studies of O and early-B supergiants reveal modest enrichment of He, whilst CNO elemental abundances have mostly been lacking until recently. Smith \& Howarth (1994) studied late OC, O and ON supergiants, revealing increasing helium abundances for these, suggesting an evolutionary sequence in support of the morphological criteria. However, great care should be taken when analysing OB stars for abundance determinations since neglect of 'micro-turbulent' line broadening may imply erroneous He-enrichments (Smith \& Howarth 1998; McErlean et al. 1998). There is clear evidence for enhanced nitrogen, accompanied with decreased carbon in OB supergiants from studies by Crowther et al. (2002), Hillier et al. (2003) and Evans et al. (2003). Notably, an ON9.7 Ia+ star in the LMC was found to be significantly more chemically processed than an otherwise identical O9.7 Ia+ star, whilst OC and BC stars were confirmed as CNO-'normal'. CNO abundances in OB supergiants in the SMC were found to exhibit similar degrees of chemical processing as A supergiants studied by Venn (1999).

\section{Further reading}

Kudritzki \& Puls (2000) have provided an excellent Annual Review article on the properties of OB stars, whilst the book by Lamers \& Cassinelli (1999) discusses Stellar Winds of both early- and late- type stars. Recent IAU Symposia involving massive stars include \#212 (van der Hucht et al. 2003) and \#215 (Maeder \& Eenens 2003).

\section{References}

Auer L.H. \& Mihalas D. 1972, ApJS 24, 193

Barlow M.J. \& Cohen M., 1977, ApJ 213, 737

Bieging J.H., Abbott D.C. \& Churchwell E.B., 1989, ApJ 340, 518

Bjorkman J.E. \& Cassinelli J.P., 1993 ApJ 409, 429

Bohannan B. \& Crowther P.A., 1999 ApJ 511, 374

Bohannan B., Abbott D.C., Voels S.A. \& Hummer D.G., 1990, ApJ 365, 729

Bouchet P., Lequeux J., Maurice E., Prevot L. \& Prevot-Burnichon M.L., 1985, A\&A 149,330

Bouret J.C. Lanz T., Hillier D.J. et al. 2003, ApJ in press (astro-ph/0301454)

Cassinelli J.P., Miller N.A., Waldron W.L., MacFarlane J.J. \& Cohen D.H., 2001, ApJ $554, \mathrm{~L} 55$

Castor J.I., Abbott D.C. \& Klein R.I., 1975, ApJ 195, 157

Collins G.W. II \& Truax, R.J., 1995, ApJ 439, 860

Conti P.S. \& Ebbets D., 1977, ApJ 213, 483

Chlebowski, T., Harnden, F. R. , Jr. \& Sciortino, S. 1989, ApJ, 341, 427

Crowther P.A. \& Dessart L., 1998, MNRAS 296, 622

Crowther P.A., Hillier D.J., Evans C.J. et al. 2002, ApJ 579, 774 
Evans C.J., Crowther P.A. et al. 2003, MNRAS in preparation

Friend D.B. \& Abbott D.C., 1986, ApJ 311, 701

Garmany C.D. \& Conti P.S., 1985, ApJ 293, 407

Georgelin Y.M. \& Georgelin Y.P, 1976, A\&A 49, 57

Gies D. 2003, in proc, IAU Symp 212, A Massive Star Odyssey, from Main Sequence to Supernova, eds. van der Hucht, K.A., Herrero A. \& Esteban C.. (San Francisco: ASP), p. 91

Gräfener G., Koesterke L. \& Hamann W-R., 2002, A\&A 387, 244

Hanson M.M., Conti P.S. \& Rieke M.J. 1996, ApJS 107, 281

Haser S.M., Pauldrach A.W.A., Lennon D.J. et al.. 1998, A\&A 330, 285

Herrero, A., Kudritzki, R. P., Vilchez, J. M., Kunze, D., Butler, K. \& Haser, S. 1992, A\&A, 261, 209

Herrero A., Puls J. \& Najarro F., 2002, A\&A 396, 949

Hilditch R.W., Harries T.J. \& Bell S.A., 1996, A\&A 314, 165

Hillier D.J. \& Miller D.L. 1998, ApJ 496, 407

Hillier D.J., Lanz T., Heap S.R. et al., 2003, ApJ 588, 1039

Howarth I.D., 1983, MNRAS, 203, 301

Howarth I.D., Siebert K.W., Hussain G.A.J. \& Prinja R.K., 1997, MNRAS 284, 265

Hubeny I. \& Lanz T., 2003, ApJS 146 in press (astro-ph/0210157)

Humphreys R.M., 1978, ApJS 38, 309

Kudritzki R.-P. \& Puls J., 2000, ARA\&A 38, 613

Kudritzki R.-P., Pauldrach A.W.A. \& Puls J., 1987, A\&A 173, 293

Kudritzki, R.-P., Pauldrach, A.W.A., Puls, J. \& Abbott, D.C. 1989, A\&A 219, 205

Kudritzki R.-P., Puls J., Lennon D.J. et al. 1999, A\&A 350, 970

Lamers H.J.G.L.M. \& Leitherer C., 1993, ApJ 412, 771

Lamers, H.J.G.L.M. \& Cassinelli J.P., 1999, Introduction to Stellar Winds, (Cambridge: CUP)

Lamers H.J.G.L.M., Cerruti-Sola M. \& Perinotto M., 1987, ApJ 314, 726

Lamers H.J.G.L.M., Snow T.P. \& Lindholm, D.M., 1995, ApJ 455, 269

Lamers H.J.G.L.M., Harzevoort J.M.A.G., Schrijver H., Hoogerwerf R. \& Kudritzki R.P., 1997, A\&A 325, L25

Leitherer C., Robert C. \& Drissen L., 1992, ApJ 401, 596

Leitherer C., Chapman J.M. \& Koribalski B., 1995, ApJ 450, 289

Lennon D.J., 1997, A\&A 317, 871

Lucy L. \& White R.L., 1980, ApJ 241, 300

Maeder A. \& Eenens P. (eds), 2003, Proc IAU Symposium 215, Stellar Rotation, (San Francisco: ASP)

Martins F., Schaerer D. \& Hillier D.J., 2002, A\&A 382, 999

Massey P., Penny L.R. \& Vukovich J., 2002, ApJ 565, 982

McErlean N.D., Lennon D.J. \& Dufton P.L., 1999, A\&A 349, 553

Mihalas D., Hummer D.G. \& Conti P.S., 1972, ApJ 175, L99

Morgan W.W., Keenan, P.C. \& Kellman E., 1943, An Atlas of Stellar Spectra with an Outline of Spectral Classification, (Chicago: UCP) 
Morton, D.C., 1967, ApJ 150, 535

Owocki S.P., Cranmer, S.R. \& Gayley K.G., 1996, ApJ 472, L115

Pauldrach A.W.A., Hoffman T.L. \& Lennon M., 2001, A\&A 375, 161

Pellerin A., Fullerton A.W., Robert C. et al. 2002, ApJS 143, 159

Petrenz P. \& Puls J., 2000, A\&A 358, 956

Pettini M., Steidel C., Adelberger K.L., Dickinson M. \& Giavalisco M., 2000, ApJ 528, 96

Plaskett J.S. \& Pearce J.S., 1931, Publ. DAO 5, 1

Prinja R.K., Barlow M.J. \& Howarth I.D., 1990, ApJ 361, 607

Prinja, R. K., \& Crowther, P. A. 1998, MNRAS, 300, 828

Puls, J., et al. 1996, A\&A, 305, 171

Runacres, M.C. \& Owocki, S.P., 2002, A\&A 381, 1015

Santaloya-Rey A.E., Puls J. \& Herrero A., 1997, A\&A 323, 488

Schaerer D. \& Schmutz W., 1994, A\&A, 288, 231

Seaton M.J., 1979, MNRAS, 187, 73

Smith L.F., Mezger, P.G. \& Biermann, P. 1978, A\&A, 66, 65

Smith K.C. \& Howarth I.D., 1998, MNRAS 299, 1146

Taresch, G., et al. 1997, A\&A, 321, 531

Vacca W.D., Garmany C.D. \& Shull J.M. 1996, ApJ, 460, 914

van der Hucht K.A., Herrero A. \& Esteban C. (eds), 2003, IAU Symposium 212: A Massive Star Odyssey, from Main Sequence to Supernova, (San Francisco: ASP)

Venn K., 1999, ApJ 518, 405

Vink J.S., de Koter A. \& Lamers H.J.G.L.M., 1999, A\&A 350, 181

Vink J.S., de Koter A. \& Lamers H.J.G.L.M., 2001, A\&A 369, 574

von Zeipel H., 1924, MNRAS 84, 665

Walborn, N.R., 1971a, ApJS 23, 257

Walborn, N.R., 1971b, ApJ 167, L31

Walborn N.R., 1976, ApJ 205, 419

Walborn, N. R. 1977, ApJ 215, 53

Walborn N.R., 2003, in proc, IAU Symp 212, A Massive Star Odyssey, from Main Sequence to Supernova, eds. van der Hucht, K.A., Herrero A. \& Esteban C., (San Francisco: ASP), p. 13

Walborn N.R., Lennon D.J., Haser S.M., Kudritzki R.-P. \& Voels S.A., 1995, PASP 107, 104

Walborn N.R., Howarth I.D., Lennom D.J. et al., 2002a AJ 123, 2754

Walborn N.R., Fullerton A.W., Crowther P.A. et al. 2002b, ApJS 141, 443

Wright A.E. \& Barlow M.J., 1975, MNRAS 170, 41 